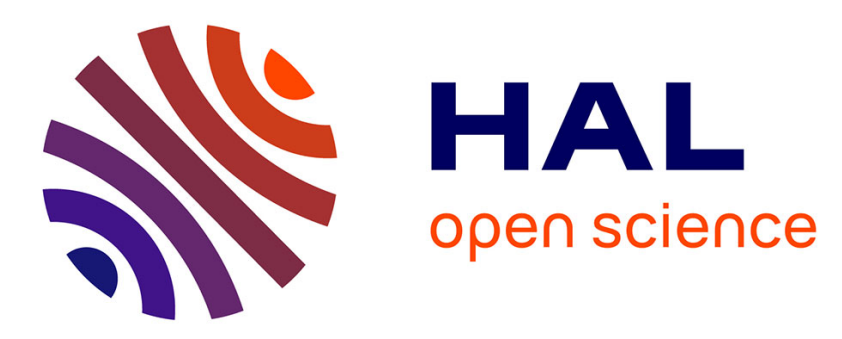

\title{
Calcul de l'énergie déposée et analyse de la cinétique d'échauffement dans ZnTe et MgZnTe sous bombardement électronique pulsé
}

\author{
C. Roussel, J.P. Jadot, B. Akamatsu, P. Henoc
}

\section{- To cite this version:}

C. Roussel, J.P. Jadot, B. Akamatsu, P. Henoc. Calcul de l'énergie déposée et analyse de la cinétique d'échauffement dans ZnTe et MgZnTe sous bombardement électronique pulsé. Revue de Physique Appliquée, 1984, 19 (3), pp.231-239. 10.1051/rphysap:01984001903023100 jpa-00245187

\section{HAL Id: jpa-00245187 https://hal.science/jpa-00245187}

Submitted on 1 Jan 1984

HAL is a multi-disciplinary open access archive for the deposit and dissemination of scientific research documents, whether they are published or not. The documents may come from teaching and research institutions in France or abroad, or from public or private research centers.
L'archive ouverte pluridisciplinaire HAL, est destinée au dépôt et à la diffusion de documents scientifiques de niveau recherche, publiés ou non, émanant des établissements d'enseignement et de recherche français ou étrangers, des laboratoires publics ou privés. 
Classification

Physics Abstracts

$34.80-34.80 \mathrm{~B}-42.55 \mathrm{R}-44.10-44.50-44.60-66.70-78.60 \mathrm{H}$

\title{
Calcul de l'énergie déposée et analyse de la cinétique d'échauffement dans ZnTe et MgZnTe sous bombardement électronique pulsé
}

\author{
C. Roussel $\left({ }^{*}\right)\left({ }^{* *}\right)$, J. P. Jadot $\left({ }^{* *}\right)$, B. Akamatsu $\left({ }^{* * *}\right)$ et P. Henoc $\left({ }^{* * *}\right)$ \\ (*) ENSIEG, Institut National Polytechnique de Grenoble, B.P. 46, 38402 St Martin d'Hères, France \\ (**) LETI, Commissariat à l'Energie Atomique, 85 X, 38041 Grenoble Cedex, France \\ ${ }^{* * *}$ ) Centre National d'Etudes des Télécommunications, 196 rue de Paris, 92220 Bagneux, France
}

(Reçu le 8 août 1983, révisé le 14 novembre, accepté le 18 novembre 1983)

\begin{abstract}
Résumé. - Corrélativement à l'étude de l'émission laser de $\mathrm{ZnTe}$ et $\mathrm{MgZnTe}$ excités par faisceau d'électrons pulsé, nous plísentons le calcul de l'énergie déposée dans ces cristaux et nous analysons la cinétique d'échauffement associée. Les profils de densité d'énergie déposée et les profondeurs de pénétration des électrons sont déterminés en simulant les trajectoires électroniques par une méthode de Monte-Carlo pour des énergies de faisceau de $15 \mathrm{keV}$ à $60 \mathrm{keV}$. La connaissance de la capacité thermique massique et de la conductivité thermique de $\mathrm{ZnTe}$ en fonction de la température permet de calculer la cinétique d'échauffement des échantillons pour des durées de quelques nanosecondes et des profondeurs de quelques micromètres. La température initiale des échantillons couvre le domaine $10 \mathrm{~K}-300 \mathrm{~K}$ et les densités de puissance étudiées sont dans la gamme $75 \mathrm{~kW} / \mathrm{cm}^{2}-1,6 \mathrm{MW} / \mathrm{cm}^{2}$. La méthode d'analyse permet de séparer dans l'excitation la contribution de l'énergie et celle de la densité de courant. Les résultats obtenus mettent en évidence un confinement thermique et montrent en particulier que l'échauffement superficiel est plus élevé à basse température en dépit d'une forte diffusion ( $\Delta T \# 40 \mathrm{~K}$ en $10 \mathrm{~ns}$ ) et que à haute température cet échauffement peut s'avérer plus important pour $15 \mathrm{keV}$ que pour $60 \mathrm{keV}$.
\end{abstract}

\begin{abstract}
In parallel with the study of laser emission in $\mathrm{ZnTe}$ and MgZnTe excited by a pulsed electron beam, we present the computation of the deposited energy in these crystals and we analyse the connected heating kinetics. The electron trajectories are simulated with a Monte-Carlo technique for beam energies from $15 \mathrm{keV}$ to $60 \mathrm{keV}$; deposited energy density profiles and penetration depths are deduced from. The variation with temperature of the specific heat and thermal conductivity of $\mathrm{ZnTe}$ leads to an analysis of heating kinetics for durations of a few nanoseconds and for depths of a few micrometers. The initial sample temperatures are studied in the range $10 \mathrm{~K}-300 \mathrm{~K}$ and the power densities in the range $75 \mathrm{~kW} / \mathrm{cm}^{2}-1.6 \mathrm{MW} / \mathrm{cm}^{2}$. This analysis permit us to distinguish between energy and current density contributions. The results indicate a thermal confinement and show that the near surface heating is larger at low temperature in spite of a strong diffusion ( $\Delta T \# 40 \mathrm{~K}$ in $10 \mathrm{~ns})$; at high temperature, this heating can be larger for $15 \mathrm{keV}$ than for $60 \mathrm{keV}$.
\end{abstract}

\section{Introduction.}

La mise en ouvre d'un banc de cathodoluminescence pulsée de forte puissance a permis de montrer l'émission laser de $\mathrm{ZnTe}$ et, pour la première fois, de $\mathrm{MgZnTe}$ [1]; dans ces expériences, le domaine utile des températures d'échantillons s'étend de moins de $10 \mathrm{~K}$ à l'ambiante. La conception d'un tel appareillage a nécessité bien évidemment d'estimer l'échauffement superficiel des échantillons sous l'excitation du faisceau d'électrons et donc d'optimiser la durée des impulsions de bombardement. Mais les récents développements de l'étude ont conduit à examiner plus précisément la cinétique de cet échauffement. Après avoir rappelé la position du problème, cet article montre comment les profils d'énergie déposée sont déterminés dans $\mathrm{ZnTe}$ et $\mathrm{MgZnTe}$ à l'aide d'une méthode de Monte-Carlo et pour des énergies de faisceau incident de $15 \mathrm{keV}$ à $60 \mathrm{keV}$. Ces profils sont ensuite utilisés pour définir le terme de génération dans l'équation de la chaleur mais en précisant les limites de validité du modèle. Un traitement numérique de cette équation permet alors de déterminer la distribution spatiale et temporelle de la température dans les échantillons (essentiellement $\mathrm{ZnTe}$ ); ceci pour des températures initiales variant de $10 \mathrm{~K}$ à $300 \mathrm{~K}$ et tout en essayant de tenir compte de l'importante évolution des caractéristiques physiques des cristaux en fonction de la température. 


\section{Position du problème.}

Ce travail correspond certes au cadre expérimental bien déterminé décrit plus précisément en [1]. Mais il peut facilement s'adapter à d'autres conditions expérimentales.

Les cristaux dont l'élaboration est détaillée en [1] sont préparés en cavités Fabry-Pérot par clivage. La figure 1 résume la géométrie et les caractéristiques dimensionnelles. Le plan à la profondeur $Z=H$ est considéré comme isothermique et à la température $T_{0}$ du porte-échantillon sur lequel les échantillons sont collés.

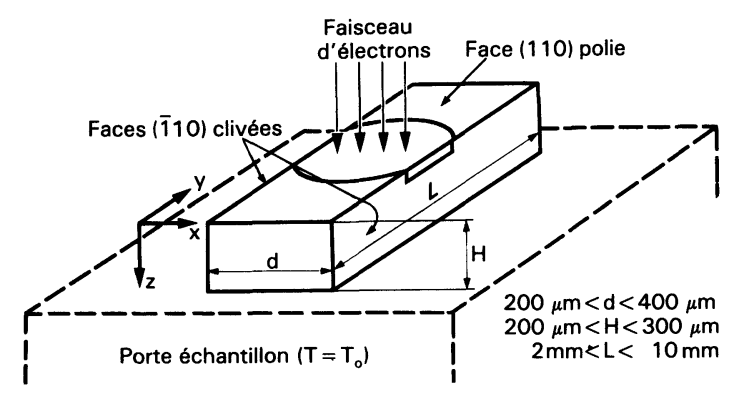

Fig. 1. - Géométrie des échantillons.

[Sample geometry.]

Tableau I. - Caractéristiques essentielles du faisceau d'électrons.

[Characteristic features of the electron beam.]

\begin{tabular}{|l|l|}
\hline $\begin{array}{c}\text { Dynamique de la tension } \\
\text { d'accélération }\end{array}$ & $0 \mathrm{kV}-60 \mathrm{kV}$ \\
\hline Domaine utile d'énergie & $15 \mathrm{keV}-60 \mathrm{keV}$ \\
\hline $\begin{array}{l}\text { Diamètre utile du spot } \\
1000 \mu \mathrm{m} \text { à } 60 \mathrm{keV}\end{array}$ \\
\hline $\begin{array}{l}\text { Densité maximale de cou- } \\
\text { rant échantillon (en im- } \\
\text { pulsions) }\end{array}$ & $27,5 \mathrm{~A} / \mathrm{cm}^{2}$ \\
\hline $\begin{array}{c}\text { Dynamique utile de la den- } \\
\text { sité de courant échantil- } \\
\text { lon }\end{array}$ & $5 \mathrm{~A} / \mathrm{cm}^{2}-27,5 \mathrm{~A} / \mathrm{cm}^{2}$ \\
\hline $\begin{array}{l}\text { Dynamique de la densité de } \\
\text { puissance }\end{array}$ & $75 \mathrm{~kW} / \mathrm{cm}^{2}-1,6 \mathrm{MW} / \mathrm{cm}^{2}$ \\
\hline $\begin{array}{l}\text { Largeur à mi-hauteur des } \\
\text { impulsions de courant } \\
\text { échantillon }\end{array}$ & $5 \mathrm{~ns}$ \\
\hline Fréquence des impulsions & $50 \mathrm{~Hz}$ \\
\hline Taux de travail & $2,5 \times 10^{-7}$ \\
\hline
\end{tabular}

Les caractéristiques essentielles du faisceau électronique sont résumées dans le tableau I. Le diamètre de la tache de focalisation de ce faisceau dépend de la haute tension mais est toujours largement supérieur à $d$. Les valeurs atteintes par la densité de puissance ont bien sûr conduit à réduire au maximum la durée des impulsions pour limiter l'échauffement instantané sous chaque tir : l'électronique de commande mise en œuvre a permis de réduire $t_{0}$, largeur à mihauteur des impulsions de courant échantillon, à une valeur aussi faible que 5 ns. Ces impulsions sont sensiblement triangulaires comme en témoigne la figure $2 \mathrm{a}$; elles correspondent en fait au fonctionnement du canon de bombardement sur une oscillation propre.

Un modèle simplifié a permis de vérifier que les caractéristiques de bombardement ainsi réalisées correspondent à un échauffement instantané du volume excité ne dépassant pas quelques degrés; soit pour le très faible taux de travail indiqué $\left(2,5 \times 10^{-7}\right)$ un échauffement moyen parfaitement
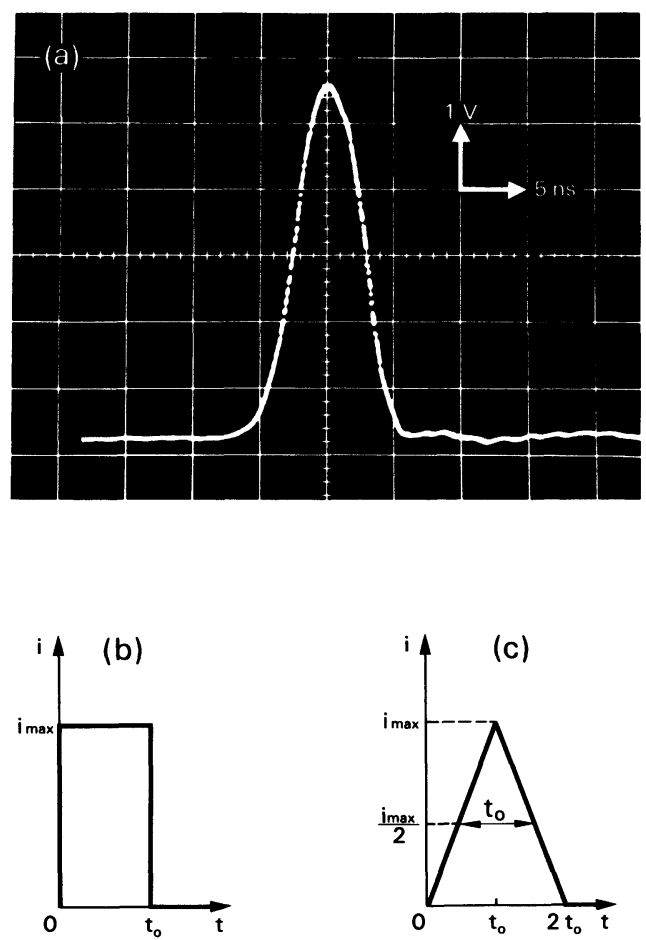

Fig. 2. - Variation du courant échantillon au cours du temps :

- Relevé expérimental (a) mesuré sur $50 \Omega$ pour $E=35 \mathrm{keV}$ (oscilloscope à échantillonnage Tektronix $564+$ amplificateur BST 6 + base de temps 3T 77A).

- Formes théoriques : abrupte (b) et triangulaire (c).

[Time variation of the sample current :

- Experimental curve (a) measured through a $50 \Omega$ load with $E=35 \mathrm{keV}$ (sampling oscilloscope Tektronix $564+$ amplifier BST 6 + time base 3T 77A).

- Theoretical curves : square (b) and triangular (c).] 
négligeable. Mais ce modèle comporte d'importantes limitations :

- la température initiale $T_{0}$ ne correspond qu'à l'ambiante et ne peut pas couvrir tout le domaine expérimental de températures car les caractéristiques physiques des échantillons sont prises constantes (et égales aux valeurs à l'ambiante);

- l'échauffement est supposé adiabatique (comme va le montrer cette étude, cette hypothèse est très raisonnable à haute température; par contre, à basse température, les mécanismes de diffusion ne peuvent pas être négligés même pour les valeurs de conductivité thermique inférieures à la valeur maximale);

- le profil d'énergie déposée est supposé abrupt avec une profondeur $Z_{\mathrm{p}}$ reposant sur des données empiriques qui ne peuvent se justifier que dans un domaine restreint d'énergie.

L'étude de la cinétique de l'émission laser a montré la nécessité de caractériser avec plus de précision l'évolution de la température superficielle des échantillons pour des durées de 2 à $3 t_{0}$, c'est-à-dire durant les quelques nanosecondes englobant l'excitation. La maîtrise de ce problème passe par une meilleure connaissance des profils d'énergie déposée dans les substrats.

\section{Détermination de l'énergie déposée dans $\mathrm{ZnTe}$ et MgZnTe.}

3.1 MÉTHODE DE DÉTERMINATION. - Les modèles mathématiques de la distribution de l'énergie déposée dans les cristaux semi-conducteurs font en général intervenir des coefficients semi-empiriques pour tenter de traduire les différents processus d'interactions électrons-matière. En général, ils ne peuvent s'appliquer que dans des domaines restreints. Bresse les a recensés, essentiellement pour le silicium [2]. Wittry et Kyser [3] indiquent une profondeur de génération de la forme $C V^{n}$. Wu et Wittry [4] utilisent une distribution d'énergie semi-gaussienne, en particulier pour GaAs.

Il est plus judicieux de déterminer les profils d'énergie déposée en utilisant la méthode qui est actuellement celle approchant le mieux les résultats expérimentaux. Elle consiste à simuler les trajectoires réelles des électrons incidents en utilisant une technique de Monte-Carlo [5-7]. Les trajectoires électroniques, à partir d'un faisceau incident ponctuel, sont schématisées figure 3. Dans un élément de trajectoire, l'électron d'énergie $E_{i}$ subit en moyenne une diffusion le long de son libre parcours moyen (déterminé à l'aide de la section efficace de diffusion de Rutherford écranté). La position A de l'événement est tirée au hasard. Et la nouvelle direction de trajectoire est définie par 2 angles tirés eux aussi au hasard : $\phi$ correspond à une distribution uniforme entre 0 et $2 \pi$; $\theta$ a une distribution associée à la fonction de répartition des sections efficaces de diffusion élastique. La perte d'énergie $E_{i}-E_{i+1}$ le long de l'élément de trajectoire est fournie par la loi de Bethe [8]. L'étape sui-
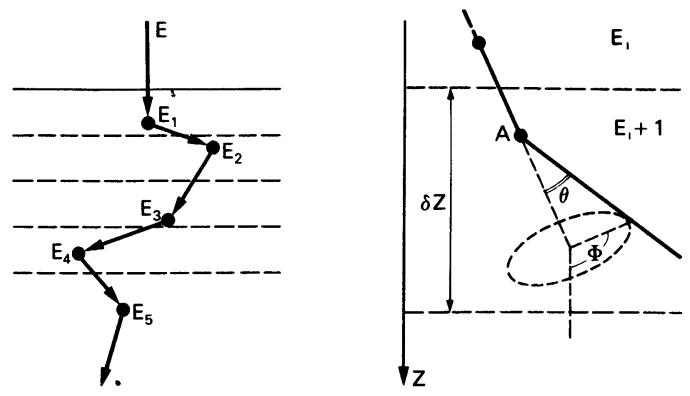

Fig. 3. - Simulation de trajectoires électroniques dans la cible.

[Simulation of electron trajectories in the target.]

vante est initialisée à partir de l'énergie $E_{i+1}$ et des nouvelles coordonnées. Il est ainsi possible de déterminer la probabilité de présence des électrons dans chaque élément de volume et de dresser alors la distribution des pertes d'énergie en profondeur (dans la direction du faisceau incident) et transversalement (perpendiculairement à l'axe du faisceau). Cette méthode fournit également le pourcentage énergétique d'électrons rétrodiffusés (c'est-à-dire d'électrons qui, après un certain parcours dans le matériau, en ressortent avec une énergie notable).

3.2 HyPOTHÈses DE TRAVAIL. - Le calcul a pu être mené pour le composé binaire $\mathrm{ZnTe}$ mais également pour le composé pseudobinaire $\mathrm{Mg}_{0,05} \mathrm{Zn}_{0,95} \mathrm{Te}$ (utilisé dans les expériences décrites en [1]). Le faisceau incident est supposé ponctuel et comporte 5000 électrons de même énergie $E$. L'étude est menée en faisant varier les valeurs de $E$ de $15 \mathrm{keV}$ à $60 \mathrm{keV}$ par pas de $5 \mathrm{keV}$. Ce calcul montre alors que l'extension radiale de la perte d'énergie reste de l'ordre de quelques micromètres. Cette extension radiale ne constitue donc qu'un effet de bord en regard de la dimension d'échantillon effectivement bombardée. Aussi, dans le contexte de la figure 1, la distribution transversale de la perte d'énergie peut parfaitement être supposée uniforme et seule la distribution en profondeur est significative : finalement dans ce qui suit, les contributions transversales de la perte d'énergie sont sommées dans chaque tranche d'épaisseur $\delta \mathrm{Z}$ parallèle à la surface.

3.3 RÉsultats. - Les résultats importants du calcul sont rassemblés dans la figure 4 qui fournit l'énergie déposée par tranche de profondeur dans $\mathrm{Mg}_{0,05} \mathrm{Zn}_{0,95} \mathrm{Te}$ et pour les différentes valeurs de l'énergie $E$. Les résultats pour $\mathrm{ZnTe}$ ne diffèrent de ceux-ci que de quelques pour-cent ; ceci était prévisible étant donnée la faible concentration de magnésium (élément léger qui plus est). Pour chaque valeur de $E$, l'énergie totale déposée dans le cristal correspond à la sommation de l'énergie déposée dans chaque tranche (Fig. 4). La différence entre l'énergie totale 

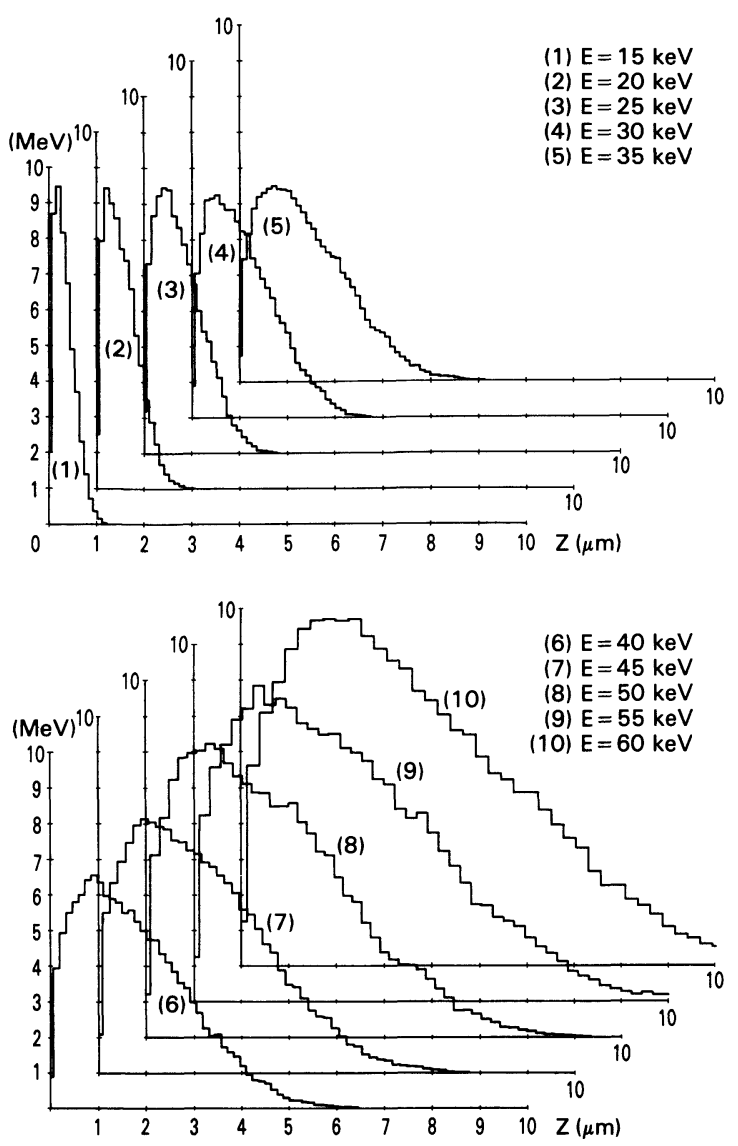

Fig. 4. - Distribution d'énergie par tranche d'épaisseur $\delta Z$ pour 5000 électrons incidents sur $\mathrm{Mg}_{0,05} \mathrm{Zn}_{0,95} \mathrm{Te}$ et en fonction de l'énergie du faisceau.

[Energy distribution in layers of thickness $\delta Z$ for 5000 incoming electrons on $\mathrm{Mg}_{0.05} \mathrm{Zn}_{0.95} \mathrm{Te}$ with different beam energies.]

incidente $(5000 \times E)$ et cette énergie totale déposée fournit la part d'énergie associée aux électrons rétrodiffusés : cette part varie approximativement de $35 \%$ pour $15 \mathrm{keV}$ à $31 \%$ pour $60 \mathrm{keV}$ (ces valeurs peuvent également être déterminées avec le programme de calcul par sommation directe de la répartition énergétique des électrons rétrodiffusés).

Enfin, chaque tranche d'épaisseur $\delta Z$ étant connue, la dérivation des courbes de la figure 4 donne immédiatement les profils de densité d'énergie déposée par unité de surface : figure 5 . Ces résultats sont importants pour la suite de cette étude. Mais ils fournissent également les valeurs de $Z_{\mathrm{p}}$ jusqu'ici indéterminées dans ces composés : $Z_{\mathrm{p}}$ est la profondeur de pénétration correspondant à la densité d'énergie moitié de la valeur maximale pour chacune des courbes de la figure 5 . Ainsi par exemple à $30 \mathrm{keV}$, $Z_{\mathrm{p}}$ se révèle être de $1,6 \mu \mathrm{m}$ dans $\mathrm{Mg}_{0,05} \mathrm{Zn}_{0,95} \mathrm{Te}$; alors qu'à la même énergie Neukermans et Saperstein [9] obtiennent dans le silicium un $Z_{\mathrm{p}}$ de $5,5 \mu \mathrm{m}$ (qui serait à comparer à la valeur obtenue ici pour $60 \mathrm{keV})$.

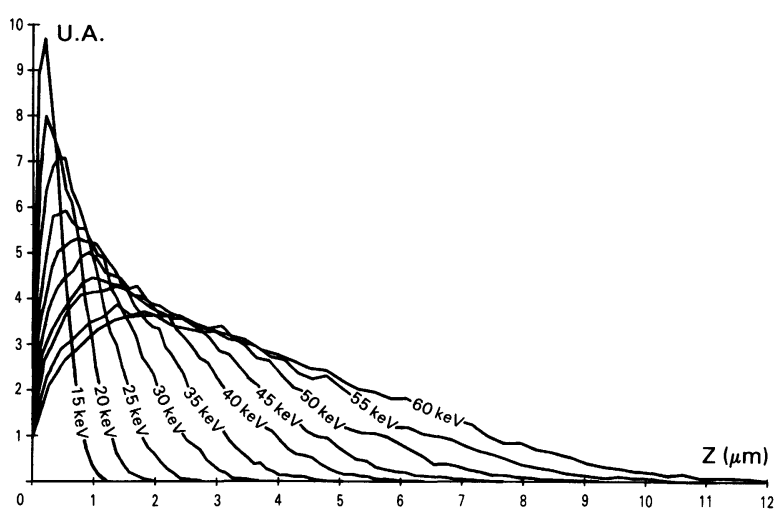

Fig. 5. - Densité d'énergie déposée dans $\mathrm{Mg}_{0,05} \mathrm{Zn}_{0,95} \mathrm{Te}$ en fonction de la profondeur et pour différentes énergies du faisceau.

[Deposited energy density in $\mathrm{Mg}_{0.05} \mathrm{Zn}_{0.95} \mathrm{Te}$ vs. depth for different beam energies.]

\section{Calcul de la cinétique d'échauffement.}

Mathématiquement, la loi de variation de la température est solution de l'équation aux dérivées partielles :

$$
\operatorname{div}(k \nabla T)=\rho C_{\mathrm{v}} \frac{\delta T}{\delta t}-G
$$

où $k$ désigne la conductivité thermique (fonction de la température $T$ ) et $\rho$ la masse volumique qui sera prise constante sur tout l'intervalle de températures étant donnée la précision visée dans ce modèle. $C_{\mathrm{v}}$ est la capacité thermique massique à volume constant; elle est très dépendante de la température mais, pour la même raison que ci-dessus, peut sans inconvénient être confondue avec $C_{\mathrm{p}}$, capacité thermique massique à pression constante.

4.1 DÉTERmination DU TAUX DE GÉNÉRATION. Le terme $G$, fonction des coordonnées $X, Y, Z$ et du temps $t$, représente le taux de génération d'énergie par unité de volume et de temps. L'hypothèse faite pour $\rho$ justifie $a$ fortiori de supposer $G$ indépendant de la température.

La distribution spatiale de $G$ correspond aux profils de densité d'énergie déposée déterminés ci-dessus. Les profils de la figure 5 sont étalonnés à partir de la densité de courant $J$ transmise dans la cible; cette méthode évite de devoir défalquer la contribution des électrons rétrodiffusés comme c'est le cas lorsque l'étalonnage est réalisé à partir de la densité de courant incident. Le modèle conduisant aux courbes de la figure 5 tient compte des électrons secondaires de forte énergie. Par contre, il néglige ceux de faible énergie malgré leur nombre relativement important et leur libre parcours moyen très faible qui les localise au voisinage des électrons primaires ; cette approximation est totalement justifiée par la distribution éner- 
gétique de ces électrons secondaires : d'une fraction d'électronvolt à quelques dizaines d'électronvolts. De même est négligée la contribution énergétique de l'émission $X$ et de l'émission lumineuse (même en régime laser). L'imprécision résultant de ces approximations ne dépasse pas quelques pour-cent et est masquée par les problèmes étudiés en 4.2.

La thermalisation des électrons primaires étant un régime nettement subnanoseconde, l'évolution temporelle de $G$ est celle du courant échantillon $i$ transmis dans la cible - ou de la densité de courant échantillon $J$. Dans un premier temps, les calculs ont été effectués avec une loi $i(t)$ abrupte correspondant à la figure $2 \mathrm{~b}$; mais les résultats indiqués ci-dessous sont plus significatifs car obtenus à partir d'une loi donnée en figure $2 c$ qui est très voisine de la forme réelle du courant (Fig. 2a).

4.2 Remarques SUR LeS Limites DE VAlidité DU MODÈLE. - La cinétique de l'échauffement qu'il est alors possible de calculer ne prend tout son sens que dans la mesure où sont utilisées les variations réelles de $k$ et de $C_{\mathrm{v}}$ en fonction de la température. Or, ces lois ne sont pas toujours bien connues pour les corps composés.

En ce qui concerne $\mathrm{ZnTe}$, la loi utilisée pour la variation de la conductivité thermique est représentée figure 6 : elle découle des mesures de Slack [10], confortées par les travaux de Bijalwan et autres [11]. La loi de variation de la capacité thermique massique est déduite des mesures de Irwin et La Combe aux températures basses et moyennes [12] ; à haute température, elle résulte d'un compromis entre l'extrapolation de ces valeurs et les données de Kelemen et autres à l'ambiante [13]; la courbe est donnée figure 7.

L'inadaptation de ces données peut constituer l'élément essentiel d'incertitude sur les résultats de ces calculs. Il peut en effet s'avérer litigieux d'utiliser directement les valeurs de $k$ et $C_{\mathrm{v}}$ compte tenu, par

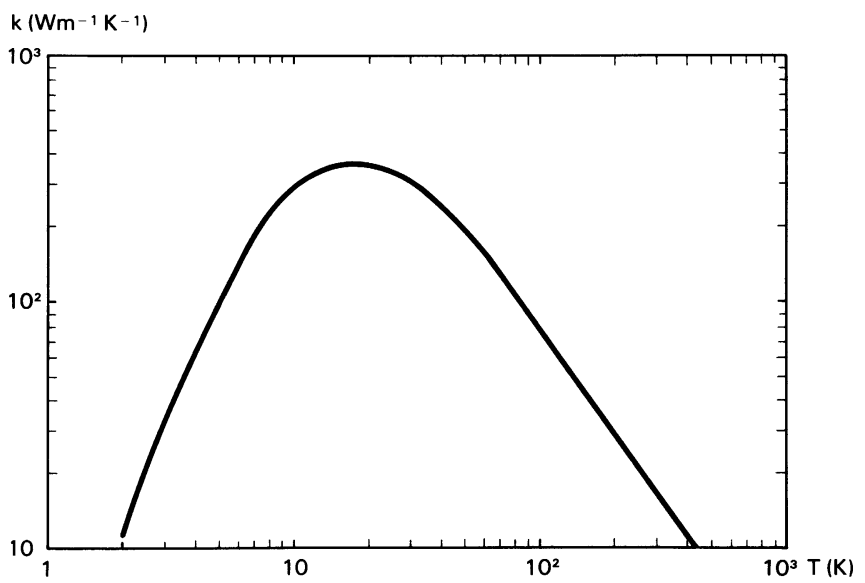

Fig. 6. - Conductivité thermique de $\mathrm{ZnTe}$ en fonction de la température : loi utilisée.

[ZnTe thermal conductivity vs. temperature (used in computations).]

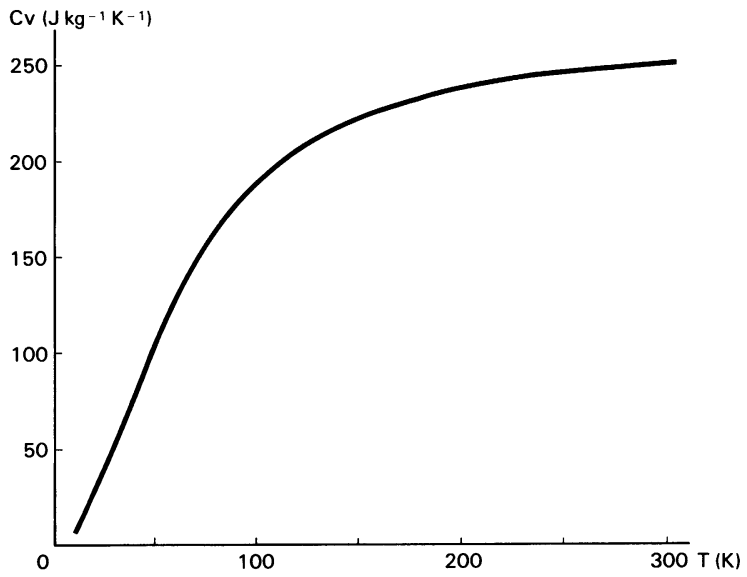

Fig. 7. - Capacité thermique massique de ZnTe en'fonction de la température : loi utilisée.

[ZnTe specific heat $v s$. temperature (used in computations).]

exemple, des problèmes de pureté chimique. Ainsi, en ce qui concerne $k$, Slack indique pour ses échantillons des concentrations d'impuretés de quelques $10^{17} \mathrm{~cm}^{-3}$; alors que, pour la majorité des échantillons utilisés ici, les concentrations d'impuretés peuvent être 10 à 100 fois plus faibles. Il est d'ailleurs à noter que - même à très basse température - la courbe de la figure 7 ne montre pas exactement une contribution de réseau correspondant à une loi de Debye en $T^{3}$.

En ce qui concerne MgZnTe, aucune valeur de $k$ ou de $C_{\mathrm{v}}$ n'a pu être trouvée et à plus forte raison pour le composé pseudobinaire $\mathrm{Mg}_{0,05} \mathrm{Zn}_{0,95} \mathrm{Te}$. Le taux de magnésium de ce composé correspond plus à un alliage quà un dopage et les valeurs ci-dessus de $k$ et de $C_{\mathrm{v}}$ ne sont vraisemblablement pas utilisables. C'est pourquoi les résultats qui suivent ne concernent que $\mathrm{ZnTe}$; et - en l'absence de mesures sur les échantillons concernés - ils ne sont transposables que qualitativement pour MgZnTe. D'après [10], il est très vraisemblable que pour ce matériau les valeurs de $k$ soient plus faibles que celles de la figure 6 . Auquel cas, surtout à basse température, les échauffements risquent d'être encore plus élevés que ceux indiqués ci-après pour $\mathrm{ZnTe}$.

D'autre part, les courbes de la figure 5 laissent prévoir que l'échauffement peut se localiser sur des profondeurs de quelques micromètres, tout au moins pour des temps restant de l'ordre de $t_{0}$ après le début de l'excitation. Cette remarque implique deux conséquences :

- Aux très basses températures, de telles profondeurs peuvent être plus faibles que le libre parcours moyen des phonons. En effet, les données ci-dessus associées à une vitesse approximative du son de $2500 \mathrm{~m} / \mathrm{s}$ (déduite soit d'une température de Debye d'environ $220 \mathrm{~K}$, soit d'une valeur moyenne de la compressibilité de l'ordre de $2,4 \times 10^{-11} \mathrm{~m}^{2} / \mathrm{N}$ d'après [14]) fournissent un libre parcours moyen des 
phonons de l'ordre de $10 \mu \mathrm{m}$ vers $10 \mathrm{~K}$. Ceci signifie qu'à cette température - et pour les durées considérées - l'équation (1) est en limite de validité et, en toute rigueur, le bilan énergétique que traduit l'équation de la chaleur devrait se traiter en termes de densité de phonons.

- Comme pour l'énergie déposée, la diffusion transversale de la chaleur ne constitue qu'un effet de bord devant l'extension du spot d'excitation. C'est pourquoi un traitement unidimensionnel se justifie pleinement.

4.3 Méthode de traitement. - Compte tenu de cette dernière remarque et en faisant abstraction des limitations indiquées, l'équation (1) est simplement traitée en symétrie plane. Le problème est discrétisé par la méthode des différences finies; puis le système linéaire résultant est résolu.

Une seule condition initiale est alors imposée : le cristal est à une température homogène $T_{0}$ au début de l'impulsion. Et les conditions aux limites se réduisent à :

— un plan isotherme (à la température $T_{0}$ ) pour $Z=H$,

- un coefficient d'échange nul à la surface de l'échantillon $(Z=0)$.

\section{Discussion des résultats.}

Les résultats du calcul peuvent être représentés sous forme de courbes isochrones de la température (ou de l'échauffement) en fonction de la profondeur $Z$. L'étude couvre toutes les températures initiales $T_{0}$, de $10 \mathrm{~K}$ à $300 \mathrm{~K}$. Les autres paramètres sont l'énergie $\mathrm{E}$ du faisceau dont 3 valeurs bien représentatives sont $15 \mathrm{keV}, 35 \mathrm{keV}$ et $60 \mathrm{keV}$ et la densité de courant échantillon $J$ dont la loi de variation est prise identique à la forme triangulaire de la figure 2c. Des courbes typiques sont groupées dans la figure 8 pour laquelle $J_{\max }$, valeur maximale de $J(t)$, est égale à la densité de courant maximale soit environ $27,5 \mathrm{~A} / \mathrm{cm}^{2}$. Ces cartes de température sont données par pas de $1 \mathrm{~ns}$ mais avec une durée limitée à 15 ns (c'est-à-dire correspondant à l'instant $3 t_{0}$ ). Suivant $Z$, l'analyse est limitée à $10 \mu \mathrm{m}$ de profondeur.

5.1 INFLUENCE DE LA TEMPÉRATURE INITIALE. - Les résultats obtenus, par exemple pour l'énergie moyenne $E=35 \mathrm{keV}$, conduisent aux remarques suivantes :

- A basse température (courbes (d) correspondant à $T_{0}=10 \mathrm{~K}$ ), il y a nettement compétition entre l'échauffement et la diffusion. Mais seul ce calcul pouvait montrer qu'en dépit d'une valeur de $k$ encore assez voisine de sa valeur maximale, la faible valeur de $C_{\mathrm{v}}$ impose un échauffement superficiel des plus importants.

- A haute température (courbes (f) : $T_{0}=300 \mathrm{~K}$ ), au contraire, la faible valeur de $k$ et la forte valeur de $C_{\mathrm{v}}$ rendent la diffusion sensiblement négligeable; l'échauffement peut presque être considéré comme adiabatique : pour les durées analysées existe un véritable confinement thermique.

- Entre ces deux extrêmes, l'évolution est continue en fonction de $T_{0}$. Le maximum de température n'est plus obtenu à la surface de l'échantillon à partir d'un $T_{0}$ supérieur à $80 \mathrm{~K}$. L'allure typique pour ces valeurs moyennes de $T_{0}$ est donnée par les courbes (e) pour $T_{0}=120 \mathrm{~K}$.

5.2 INFLUENCE DE L'ÉNERGIE. - L'ensemble des courbes obtenues, toujours pour cette densité de courant maximale, montre également l'influence de l'énergie des électrons incidents :

- Aux énergies élevées, la valeur de $Z_{\mathrm{p}}$ est telle que l'échauffement n'est pas plus élevé à $60 \mathrm{keV}$ qu’à $35 \mathrm{keV}$ pour $T_{0}=10 \mathrm{~K}$ (courbes (g)); aux hautes températures, il est même plus faible pour $60 \mathrm{keV}$ bien que le confinement thermique soit net (courbes $(\mathrm{h})$ et (i)).

- Aux faibles énergies, l'échauffement est bien sûr plus faible que ci-dessus pour les faibles valeurs de $T_{0}$ comme le montrent les courbes (a) pour $15 \mathrm{keV}$ et $10 \mathrm{~K}$; mais pour $T_{0}$ supérieur à $50 \mathrm{~K}$, il ne varie pratiquement plus; et à haute température (courbes (b) et (c)), le confinement est tel que l'échauffement est plus élevé que celui correspondant aux fortes énergies. Ce résultat, difficilement prévisible, n'aurait pu être mis en évidence aussi simplement si, comme cela se fait habituellement, l'analyse avait été menée en qualifiant l'excitation en terme de densité de puissance au lieu de séparer la contribution de l'énergie et celle de la densité de courant.

5.3 Influence de la densité de Courant. - Des courbes semblables à celles de la figure 8 sont obtenues pour d'autres valeurs de la densité de courant échantillon, en particulier pour $J_{\max }=5 \mathrm{~A} / \mathrm{cm}^{2}$ correspondant à l'autre extrême de la dynamique utile pour $J$. Les résultats ne montrent pas de modification importante quant à la forme générale des courbes : seules les amplitudes d'échauffement diffèrent comme il est indiqué en figure 9.

5.4 EXPLOITATION DES RÉSUltats. - Tous ces résultats peuvent être rassemblés sous des formes plus pratiques. Le paramètre essentiel à étudier est bien sûr l'échauffement maximum en fonction de $T_{0}$, pour différentes énergies et densités de courant. Sa variation est représentée figure 9. La position en profondeur de cet échauffement maximum est également informative; dans une large gamme de température et pour $J_{\max }=27,5 \mathrm{~A} / \mathrm{cm}^{2}$, elle correspond à $Z=0$ pour $15 \mathrm{keV}, Z \# 1 \mu \mathrm{m}$ pour $35 \mathrm{keV}$ et $Z \# 2 \mu \mathrm{m}$ pour $60 \mathrm{keV}$.

Enfin, il est utile de qualifier le confinement thermique : la méthode retenue consiste à déterminer la profondeur $Z_{\mathrm{T}}$ pour laquelle un échauffement moitié de l'échauffement maximum est obtenu au temps $t=2 t_{0}$ (c'est-à-dire à la fin de l'impulsion triangulaire) : les variations en sont représentées figure 10. 


\section{$15 \mathrm{keV}$}
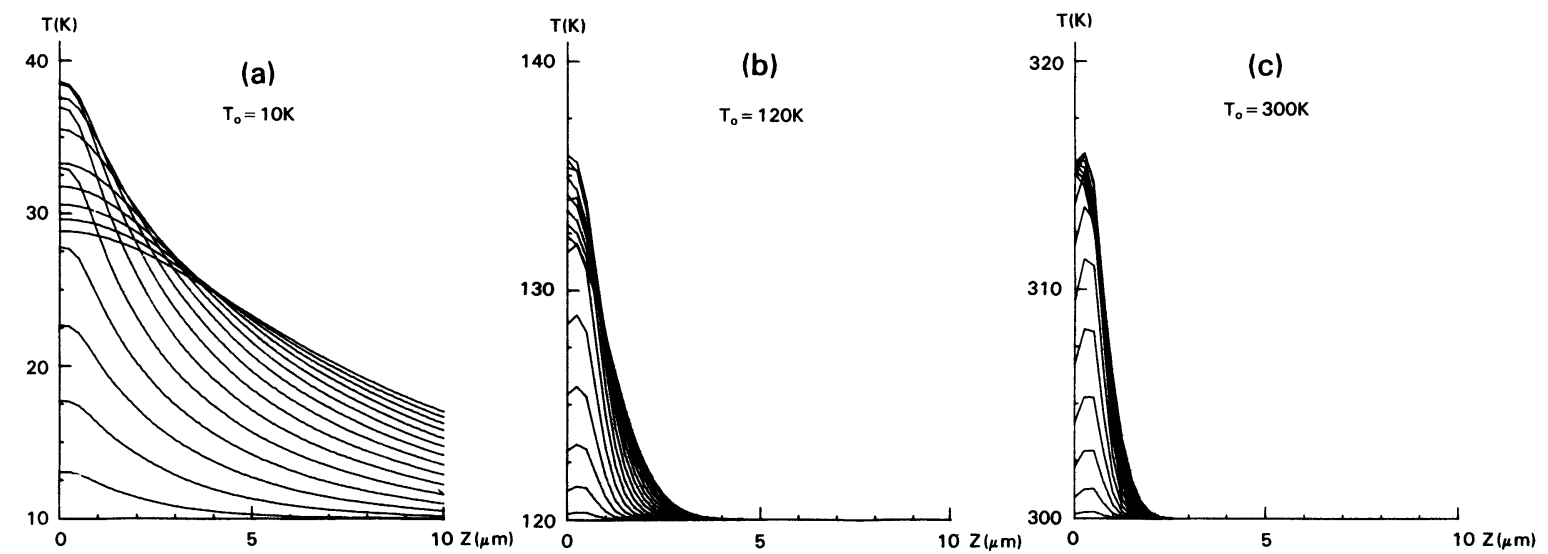

$35 \mathrm{keV}$
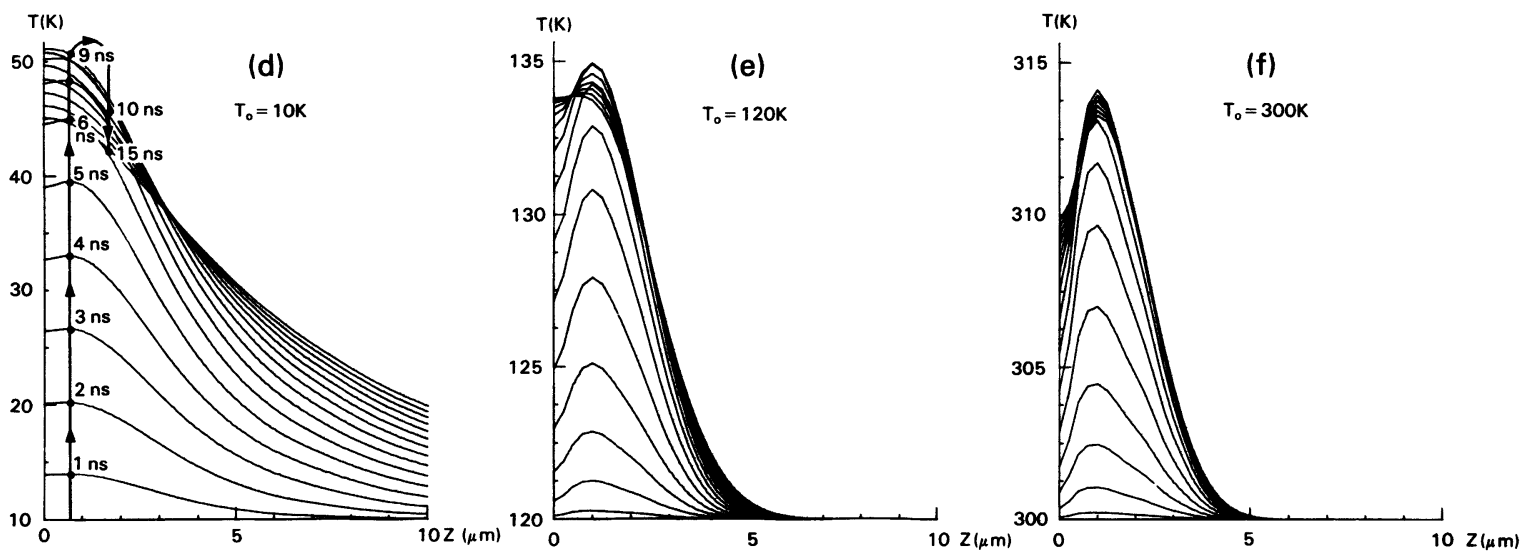

$60 \mathrm{keV}$
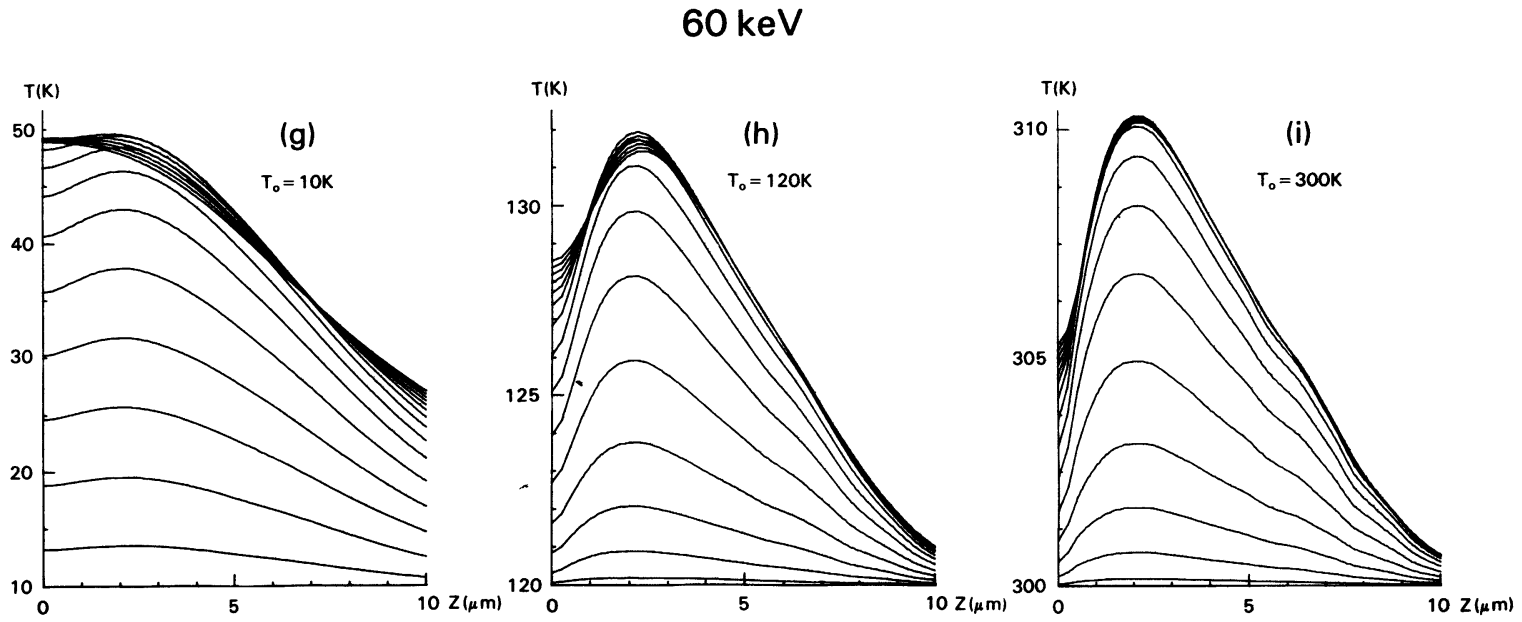

Fig. 8. - Isochrones de température dans $\mathrm{ZnTe}$ (par pas de $1 \mathrm{~ns}$ jusqu'à $15 \mathrm{~ns}$ ) en fonction de la profondeur (jusqu'à $10 \mu \mathrm{m}$ ) pour 3 énergies de faisceau $E$ et 3 températures de substrat $T_{0}$. La densité de courant échantillon correspond à la loi triangulaire de la figure $2 \mathrm{c}$ avec $J_{\max }=27,5 \mathrm{~A} / \mathrm{cm}^{2}$.

[Curves of $\mathrm{ZnTe}$ temperature at constant times (from 0 to $15 \mathrm{~ns}$ by $1 \mathrm{~ns}$ ) vs. depth (down to $10 \mu \mathrm{m}$ ) for 3 different beam energies $E$ and 3 substrate temperatures $T_{0}$. The sample current density is taken as triangular with $J_{\max }=27.5 \mathrm{~A} / \mathrm{cm}^{2}$.] 


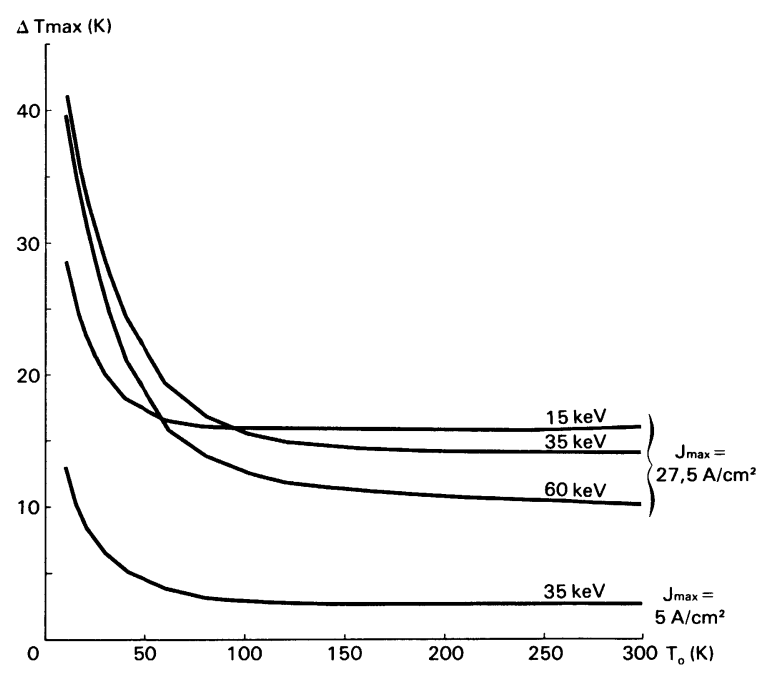

Fig. 9. - Echauffement maximum dans $\mathrm{ZnTe}\left(\Delta T_{\max }=\right.$ $T_{\max }-T_{0}$ ) en fonction de $T_{0}$ pour 3 énergies différentes et 2 densités de courant échantillon.

[Maximal heating in $\operatorname{ZnTe}\left(\Delta T_{\max }=T_{\max }-T_{0}\right) v s . T_{0}$ for 3 different energies and 2 sample current densities.]

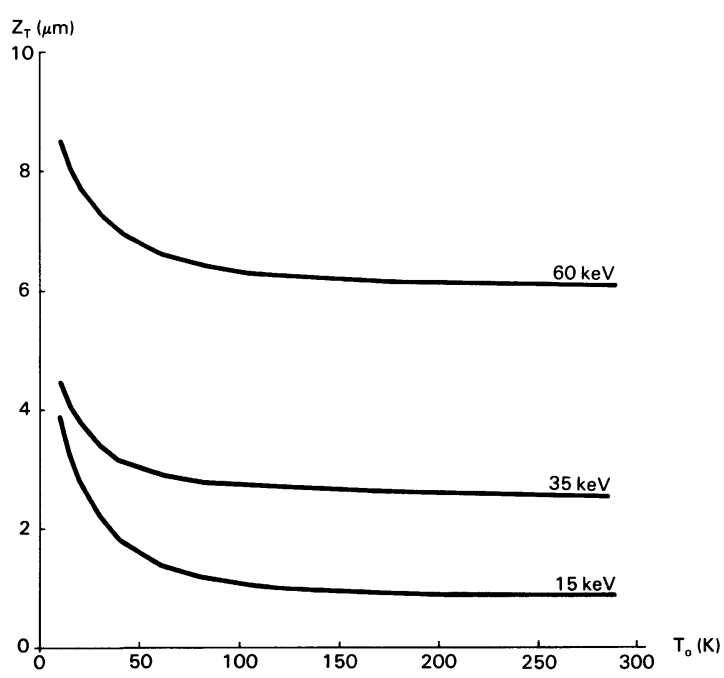

Fig. 10. - Evolution de $Z_{\mathrm{T}}$ pour différentes énergies dans $\operatorname{ZnTe}\left(Z_{\mathrm{T}}=Z\right.$ pour $T=T_{0}+\frac{\Delta T_{\max }}{2}$ et $\left.t=2 t_{0}\right)$.

$\left[Z_{\mathrm{T}}\right.$ variation in $\mathrm{ZnTe} v s . T_{0}$ for different energies $\left(Z_{\mathrm{T}}=Z\right.$ for $T=T_{0}+\frac{\Delta T_{\max }}{2}$ et $\left.t=2 t_{0}\right)$.]

5.5 CinÉTIQUe de L'ÉCHAUFFEMENT. - Elle constitue l'autre aspect important des résultats obtenus. La figure 11 donne la variation dans le temps de la température de surface $(Z=0)$ pour différentes énergies et pour $T_{0}=10 \mathrm{~K}$, avec $J_{\max }=27,5 \mathrm{~A} / \mathrm{cm}^{2}$.

L'échauffement maximum est atteint soit dans les 2 nanosecondes précédant la fin de l'impulsion (à basse température), soit exactement à la fin de l'im-

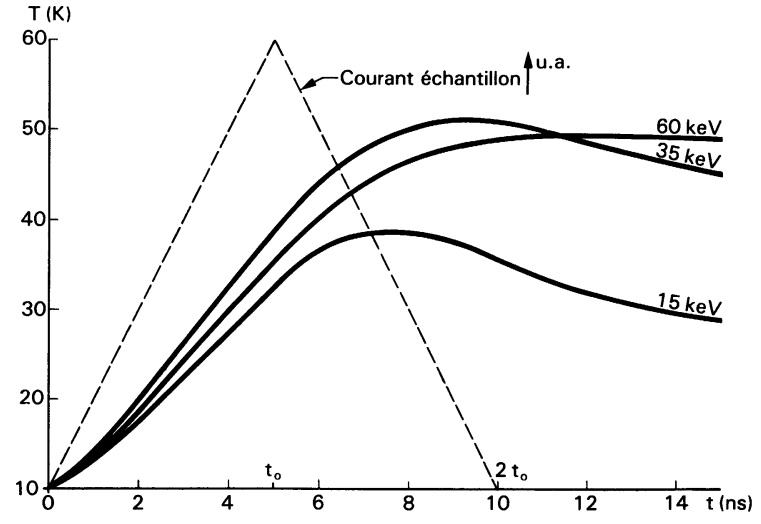

Fig. 11. - Variation de la température de surface de $\mathrm{ZnTe}$ durant les 15 premières nanosecondes pour différentes énergies et $T_{0}=10 \mathrm{~K}$ avec $J_{\max }=27,5 \mathrm{~A} / \mathrm{cm}^{2}$.

[ZnTe surface temperature variation during the 15 first nanoseconds, for different energies; $T_{0}=10 \mathrm{~K}$ and $J_{\max }=27.5 \mathrm{~A} / \mathrm{cm}^{2}$.]

pulsion (à haute température). Par contre, le maximum de température à la surface, lui, peut être atteint pour des temps grands devant $t_{0}$ (c'est toujours le cas à haute température).

Sur des durées beaucoup plus longues, il est aisé de vérifier que l'échantillon retrouve sa température initiale $T_{0}$ en des temps variant de quelques dizaines de microsecondes (à basse température) à quelques centaines de microsecondes (à haute température). Ceci justifie pleinement la fréquence des impulsions $(50 \mathrm{~Hz})$ et le fait d'avoir mené tous ces calculs pour une impulsion unique.

\section{Conclusion.}

Ce travail a permis, par une méthode de MonteCarlo, de déterminer les profils de densité d'énergie déposée dans $\mathrm{ZnTe}$ et $\mathrm{MgZnTe}$ par bombardement électronique. L'analyse est menée pour des énergies de faisceau allant de $15 \mathrm{keV}$ à $60 \mathrm{keV}$. Il est montré que les géométries de spot et d'échantillon justifient de traiter le modèle en symétrie plane. Moyennant certaines hypothèses sur la conductivité thermique et la capacité thermique massique, une étude thermocinétique du régime pulsé a été menée - pour $\mathrm{ZnTe}$ tout au moins. Les températures initiales d'échantillon vont de $10 \mathrm{~K}$ à $300 \mathrm{~K}$. L'échauffement des échantillons est caractérisé pour des profondeurs et des durées correspondant à celles concernées par l'émission laser : $10 \mu \mathrm{m}$ et $10 \mathrm{~ns}$. La méthode d'analyse a montré séparément l'influence de la densité de courant échantillon et celle de l'énergie du faisceau d'excitation.

Les résultats obtenus permettent de comparer la profondeur $Z_{\mathrm{T}}$ - qualifiant le confinement thermique - à la profondeur $Z_{\mathrm{p}}$ de pénétration de l'énergie. A haute température, les $Z_{\mathrm{T}}$ sont sensiblement constants mais supérieurs aux $Z_{\mathrm{p}}$. Et, bien que la diffusion 
soit peu notable, l'échauffement maximum ne dépasse guère quelques degrés ou la dizaine de degrés suivant l'énergie. A basse température au contraire, la diffusion de chaleur est importante $\left(Z_{\mathrm{T}}\right.$ grand); mais malgré tout, l'échauffement maximum peut atteindre plusieurs dizaines de degrés en quelques nanosecondes.
Ces échauffements sont en bon accord avec les résultats observés sur les spectres de luminescence. Cette cinétique d'échauffement, associée à la cinétique de l'émission laser, doit permettre une bonne interprétation des spectres obtenus en régime laser avec ces matériaux.

\section{Bibliographie}

[1] Roussel, C. et Ermolieff, A., Revue Phys. Appl. 18-1 (1983) 1.

[2] Bresse, J. F., Thèse Docteur Ingénieur, Université Scientifique et Médicale de Grenoble (1974).

[3] Wittry, D. B. et Kyser, D. F., J. Appl. Phys. 38-1 (1967) 375.

[4] Wu, C. J. et Wittry, D. B., J. Appl. Phys. 49-5 (1978) 2827.

[5] Henoc, J. et Maurice, F., Rapport du Commissariat à l'Energie Atomique, CEA R 4615 (1975).

[6] Henoc, J. et MAURiCE, F., Rapport National Bureau of Standards, DC, 460 (1976) 61

[7] Akamatsu, B., Henoc, J. et Henoc, P., J. Micros Spectros. Electron. 2 (1977) 635.
[8] Bethe, M. A., Ann. Physik 5 (1930) 325.

[9] Neukermans, A. et Saperstein, W., J. Vac. Sci. Technol. 16-6 (1979) 1847.

[10] Slack, G. A., Phys. Rev. B 6-10 (1972) 3791.

[11] Bijalwan, R. D., Ram, P. N. et Tiwari, M. D., J. Phys. C 16 (1983) 2537.

[12] Irwin, J. C. et LA Combe, J., J. Appl. Phys. 45-2 (1974) 567.

[13] Kelemen, F., Cruceanu, E. et Niculescu, D., Phys. Status Solidi 11-2 (1965) 865.

[14] Cline, C. G. et Stephens, D. R., J. Appl. Phys. 35-9 (1965) 2869. 